GA-A22729

$$
\text { CONF-971065-- }
$$

\title{
MULTIVARIABLE SHAPE CONTROL DEVELOPMENT ON THE DIII-D TOKAMAK
}

RECEIVE JAN 261998 OSTI

by
M.L. WALKER, D.A. HUMPHREYS, and J.R. FERRON

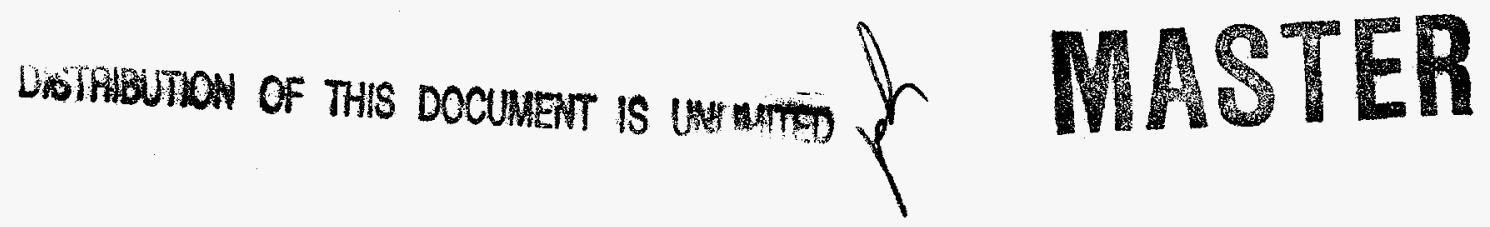

NOVEMBER 1997 
Table A2. Annual U.S. Macroeconomic and Weather Indicators

\begin{tabular}{|c|c|c|c|c|c|c|c|c|c|c|c|c|c|c|c|}
\hline & \multicolumn{15}{|c|}{ Year } \\
\hline & 1985 & 1986 & 1987 & 1988 & 1989 & 1990 & 1991 & 1992 & 1993 & 1994 & 1995 & 1996 & 1997 & 1998 & 1999 \\
\hline \multicolumn{16}{|l|}{ Macroeconomic } \\
\hline 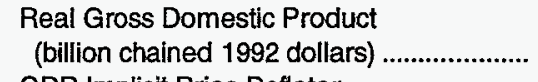 & 5324 & 5488 & 5649 & 5865 & 6062 & 6136 & 6079 & 6244 & 6390 & 6611 & 6742 & 6928 & 7187 & 7345 & 7465 \\
\hline $\begin{array}{l}\text { GDP Implicit Price Deflator } \\
\text { (Index, 1992=1.000) }\end{array}$ & 0786 & & & & & & & & & & & & & & \\
\hline Real Disposable Personal Income & 0.00 & 0.000 & 1 & 8.00 & 0.097 & 0.930 & 0.973 & 1.000 & 1.020 & 1.051 & 1.078 & 1.102 & 1.125 & 1.144 & 1.164 \\
\hline 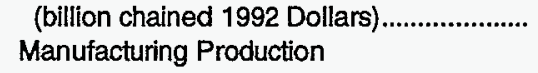 & 3972 & 4101 & 4168 & 4332 & 4417 & 4498 & 4500 & 4627 & 4704 & 4805 & 4964 & 5077 & 5220 & 5398 & 5519 \\
\hline $\begin{array}{l}\text { (Index, } 1987=1.000) \\
\text { Real Fixed Investment }\end{array}$ & 0.857 & 0.881 & 0.928 & 0.971 & 0.990 & 0.985 & 0.962 & 1.000 & 1.037 & 1.094 & 1.132 & 1.164 & 1.224 & 1.262 & 1.272 \\
\hline $\begin{array}{l}\text { (billion chained } 1992 \text { dollars) .................... } \\
\text { Real Exchange Rate }\end{array}$ & 799 & 805 & 799 & 818 & 832 & 806 & 741 & 783 & 843 & 916 & 962 & 1042 & 1125 & 1201 & 1234 \\
\hline $\begin{array}{l}\text { (Index, } 1990=1.000) \\
\text { Business Inventory Change }\end{array}$ & NA & NA & NA & NA & NA & 1.000 & 1.006 & 1.012 & 1.056 & 1.033 & 0.960 & 1.015 & 1.097 & 1.086 & 1.050 \\
\hline $\begin{array}{l}\text { (billion chained } 1992 \text { dollars) ..................... } \\
\text { Producer Price Index }\end{array}$ & -4.5 & -4.2 & 5.1 & 9.5 & 19.2 & 6.6 & -6.1 & -9.2 & 6.1 & 11.1 & 7.8 & 9.9 & 20.2 & 4.7 & -3.4 \\
\hline $\begin{array}{l}\text { (index, 1980-1984=1.000) } \\
\text { Consumer Price Index....................... }\end{array}$ & 1.032 & 1.002 & 1.028 & 1.069 & 1.122 & 1.163 & 1.165 & 1.172 & 1.189 & 1.205 & 1.248 & 1.277 & 1.275 & 1.276 & 1.286 \\
\hline $\begin{array}{l}\text { (index, 1980-1984=1.000) } \\
\text { Petroleum Product Price Index }\end{array}$ & 1.076 & 1.097 & 1.137 & 1.184 & 1.240 & 1.308 & 1.363 & 1.404 & 1.446 & 1.483 & 1.525 & 1.570 & 1.606 & 1.634 & 1.668 \\
\hline 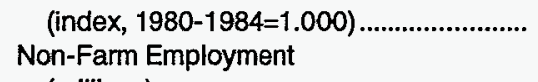 & 0.832 & 0.532 & 0.568 & 0.539 & 0.612 & 0.748 & 0.671 & 0.647 & 0.620 & 0.591 & 0.608 & 0.701 & 0.679 & 0.611 & 0.624 \\
\hline 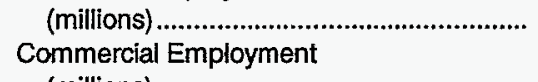 & 97.4 & 99.3 & 102.0 & 105.2 & 107.9 & 109.4 & 108.3 & 108.6 & 110.7 & 114.1 & 117.2 & 119.5 & 122.2 & 124.6 & 126.0 \\
\hline $\begin{array}{l}\text { (millions) } \\
\text { Total Industrial Production }\end{array}$ & 60.8 & 62.9 & 65.2 & 67.8 & 70.0 & 71.3 & 70.8 & 71.2 & 73.2 & 76.1 & 78.8 & 81.0 & 83.5 & 85.5 & 86.9 \\
\hline $\begin{array}{l}\text { (index, } 1987=1.000) \\
\text { Housing Stock }\end{array}$ & 0.880 & 0.890 & 0.931 & 0.973 & 0.990 & 0.989 & 0.969 & 1.000 & 1.034 & 1.086 & 1.121 & 1.152 & 1.206 & 1.239 & 1.249 \\
\hline 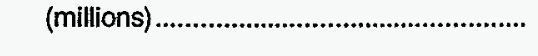 & 96.3 & 98.0 & 99.8 & 101.6 & 102.9 & 103.5 & 104.5 & 105.5 & 106.8 & 108.2 & 109.8 & 111.2 & 112.7 & 114.2 & 115.6 \\
\hline $\begin{array}{l}\text { Weather } \\
\text { Heating Degree-Days }\end{array}$ & & & & & & & & & & & & & & & \\
\hline U.S. & 4642 & 4295 & 4334 & 4653 & 4726 & 4016 & 4200 & 4441 & 4700 & 4483 & 4531 & 4713 & 4675 & 4576 & 4576 \\
\hline 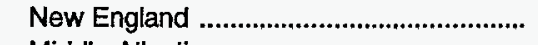 & 6571 & 6517 & 6546 & 6715 & 6887 & 5848 & 5960 & 6844 & 6728 & 6672 & 6559 & 6679 & 6894 & 6621 & 6621 \\
\hline 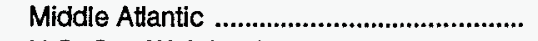 & 5660 & 5665 & 5699 & 6088 & 6134 & 4998 & 5177 & 5964 & 5948 & 5934 & 5831 & 5986 & 6040 & 5839 & 5839 \\
\hline 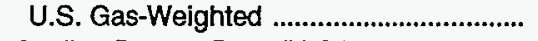 & 4856 & 4442 & 4391 & 4779 & 4856 & 4139 & 4337 & 4458 & 4754 & 4659 & 4707 & 5040 & 4912 & 4732 & 4732 \\
\hline 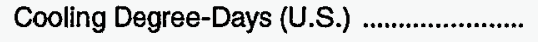 & 1194 & 1249 & 1269 & 1283 & 1156 & 1260 & 1331 & 1040 & 1218 & 1220 & 1293 & 1180 & 1091 & 1193 & 1193 \\
\hline
\end{tabular}

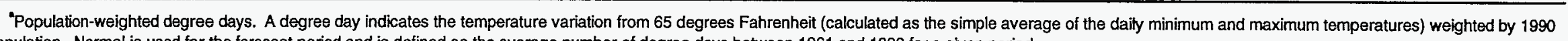

population. Normal is used for the forecast period and is defined as the average number of degree days between 1961 and 1990 for a given period.
Notes: Historical data are printed in bold; forecasts are in italics.

Sources: Historical data: latest data available from: U.S. Department of Commerce, Bureau of Economic Analysis; U.S. Department of Commerce, National Oceanic and Atmospheric Administration; Federal Reserve System, Statistical Release G.17(419); U.S. Department of Transportation; American Iron and Steel Institute. Macroeconomic projections are based on DRIMcGraw-Hill Forecast CONTROL1297. 


\section{DISCLAIMER}

Portions of this document may be illegible electronic image products. Images are produced from the best available original document. 


\title{
MULTIVARIABLE SHAPE CONTROL DEVELOPMENT ON THE DIII-D TOKAMAK
}

\author{
by \\ M.L. WALKER, D.A. HUMPHREYS, and J.R. FERRON
}

This is a preprint of a paper to be presented at the 17th IEEE/NPSS Symposium on Fusion Engineering, October 6-11, 1997, San Diego, California and to be published in the Proceedings.

\author{
Work supported by \\ the U.S. Department of Energy \\ under Contract No. DE-AC03-89ER51114
}




\title{
Multivariable Shape Control Development on the DIII-D Tokamak*
}

\author{
M.L. Walker, D.A. Humphreys, and J.R. Ferron \\ General Atomics \\ P.O. Box 85608, San Diego, California 92186-9784
}

\begin{abstract}
In this paper, we describe recent work on plasma shape and position control at DIII-D. This control consists of two equally challenging problems - the problem of identifying what the plasma actually looks like in real time, i.e. measuring the parameters to be controlled, and the task of determining the feedback algorithm which best controls these plasma parameters in a multiple input-output system. Recent implementation of the EFIT plasma equilibrium reconstruction algorithm in real time code-which produces a new equilibrium estimate every $1.5 \mathrm{~ms}$ seems to solve the longstanding problem of obtaining sufficiently accurate plasma shape and position estimation. Stabilization of the open-loop unstable vertical motion is also viewed as a solved problem. The primary remaining problem appears to be how best to command the power supplies to achieve a desired shaping control response. We will describe our effort to understand and apply linearized models of plasma evolution to development and implementation of multivariable plasma controllers.
\end{abstract}

\section{INTRODUCTION}

A recent change of control methodology at DIII-D has been the transition from so-called "gap control" [1] to "isoflux" control [2] which exploits the capability of the new real time EFIT algorithm to calculate magnetic flux at specified locations within the tokamak vessel. Fig. 1 illustrates a lower single null plasma which was controlled using the new isoflux control. Real time EFIT can calculate very accurately the value of flux in the vicinity of the plasma boundary. Thus, the controlled parameters are the values of flux at prespecified control points along with the $\mathrm{X}$-point $\mathrm{r}$ and $\mathrm{z}$ position. By requiring that the flux at each control point be equal to the same constant value, the control forces the same flux contour to pass through all of these control points. By choosing this constant value equal to the flux at the $\mathrm{X}$-point, this flux contour must be the last closed flux surface or separatrix. The desired separatrix location is specified by selecting one of a large number of control points along each of several control segments (Fig. 1). An X-point control grid is used to assist in calculating the $\mathrm{X}$-point location by providing detailed flux and field information at a number of closely spaced points in the vicinity of the $\mathrm{X}$-point.

\section{DIII-D CONTROL MODELS}

To develop a multivariable controller for poloidal shaping, it is necessary to develop and validate a model of the system(s)

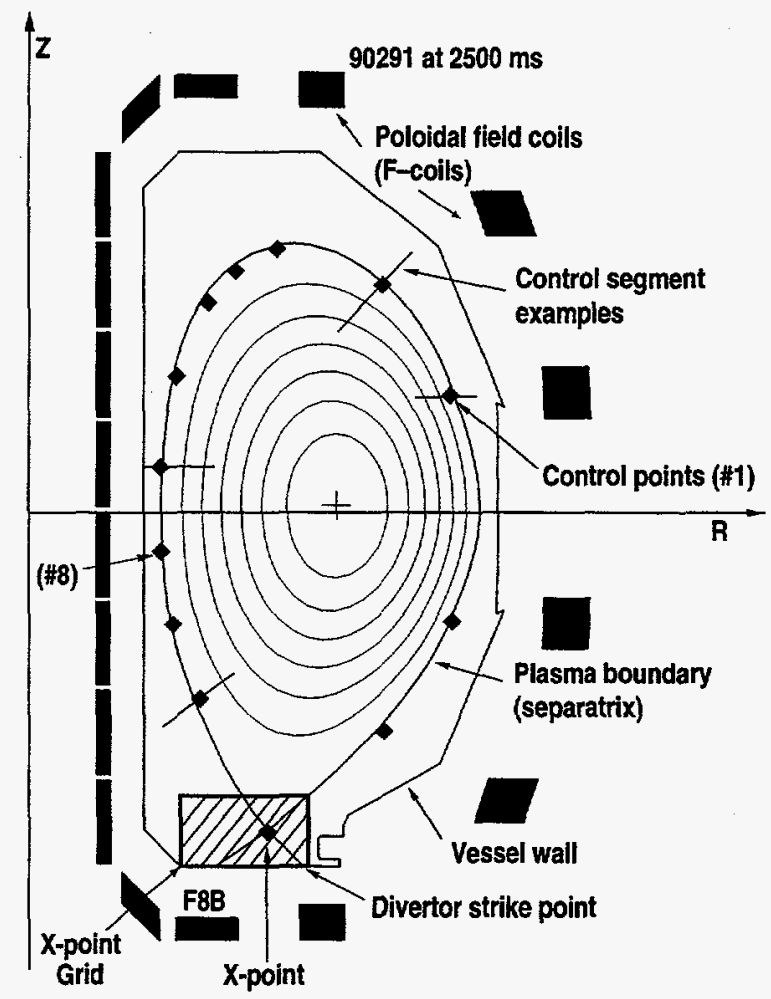

Fig. 1. Example of controlled plasma parameters in new isoflux control $\left(R_{X}\right.$, $Z_{X}$, and flux at control points \#1-13 on control segments).

to be controlled. Models of these systems include a highly nonlinear plasma, a large but linear set of circuit equations defining shaping currents as a function of applied voltage, and shaping power supplies which also require highly nonlinear models.

Knowledge of DIII-D conductor resistances and mutual inductances are used to construct the circuit model $M_{d d} d I_{d} / d t+R_{d} I_{d}=V_{d}$ of external toroidal conductors (i.e. those conductors which influence poloidal shape) in DIII-D. Here, $M_{d d}$ and $R_{d}$ are the conductor-to-conductor mutual inductance matrix and the (diagonal) conductor resistance matrix, both modified by circuit loop connections, $I_{d}$ is the vector of currents in all conductors, and $V_{d}$ is the vector of voltages in series with the Ohmic heating coil (E-coil) and poloidal shaping coils (F-coils). This model has been extensively validated [3].

The plasma response consists of the variations in flux at

\footnotetext{
*Work supported by U.S. Department of Energy under Contract No. DE-AC03-89ER51114.
} 
conductors resulting from plasma shape changes. The plasma shape in turn varies in response to conductor currents. The linearized effect of the plasma on the conductor dynamics can therefore be modeled as an additional mutual inductance term in the conductor circuit equation, which becomes $M_{d d} \dot{I}_{d}+R_{d} I_{d}+X_{d d} \dot{I}_{d}=V_{d}$. In this equation, $X_{d d}$ describes flux variations at conductors due to plasma shape and position changes which in turn arise from conductor current changes. The response of the plasma to coil currents $\left(X_{d d}\right)$ can contribute variations in flux at conductors comparable to flux variations from conductor currents alone $\left(M_{d d}\right)$. In particular, variations in conductor flux resulting from vertical motion of the plasma are dominated by the plasma contribution, giving rise to the only unstable mode of the system, known as the "vertical instability". Use of a plasma response model is thus necessary at some level to produce an efficient stabilizing controller, and can in principle improve control of the remaining stable modes.

We wish to find simple, generally applicable models to reduce the complexity of a resulting control system. In particular, we would like to avoid gain scheduling a large number of high degree controllers in a single plasma discharge. The least complex option would be if we were able to design controllers without reference to a plasma model. Failing that, crude models which were appropriate over many different plasmas would be preferred.

The first plasma model we consider here is a crude one degree of freedom approximation in which plasma current is concentrated in a single filament at the plasma current centroid and only rigid radial plasma motion is allowed. We also consider below a single filament model allowing both radial ( $\mathrm{r}$ ) and vertical (z) rigid motion. One comparison we can make with the no-plasma-model case is the following: each linearized model describing the response $y$ of flux at control points and field at the $X$-point is given by a state space description of the form $d \mathrm{I} / \mathrm{dt}=\mathrm{AI}+\mathrm{BV}, \mathrm{y}=\mathrm{CI}+\mathrm{DV}$, with the comparison

$$
\begin{aligned}
& A_{\text {rrig }}=A_{\text {nopl }}+\Delta A,\|\Delta A\| /\left\|A_{\text {nopl }}\right\|=0.0399 \\
& B_{\text {rrig }}=B_{\text {nopl }}+\Delta B, \quad\|\Delta B\| /\left\|B_{\text {nop } 1}\right\|=0.0025 \\
& C_{\text {rig }}=C_{\text {nopl }}+\Delta C,\|\Delta C\| / /\left\|C_{\text {nop }}\right\|=1.3985 \\
& D_{\text {rrig }}=D_{\text {nopl }}=0,
\end{aligned}
$$

where the "rrig" subscript indicates that the system includes effects due to the rigid radial plasma, "nopl" indicates it does not. Here $\Delta \mathrm{C}$ models the change in flux and field from motion of the plasma current filament which is due to changes in conductor current. Note that the dynamics of the current evolution in the external conductors will likely not be much different with or without a "rigid radial" plasma present. The flux and field response to conductor currents is significantly different, however.
Thus one key test of a good plasma response model is accuracy with which we can predict flux and field changes due to changes in conductor currents. To this end, an experiment was conducted in which one coil at a time was stepped by a few hundred Amperes from the equilibrium current value. All other coil currents were held constant. Because of hardware constraints imposed on the relationship between coil currents, they were not constant but were relatively smaller than the chosen coil. The single filament "rigid $r$," "rigid $r$ and $z$," and vacuum (no-plasma) models were used for comparison. For purposes of isoflux control, we want to be able to predict changes in flux at control points and changes in the $\mathrm{X}$-point location due to changes in F-coil currents. Fig. 2 shows some sample results. The vacuum model produces much less variability than the plasma models and thus appears constant.

We are also interested in the accuracy of simple models in prediction of the plasma growth rate for the unstable vertical motion, although this is not as important as the flux and $\mathrm{X}$-point prediction because we already have a satisfactory vertical position stabilizing controller [4]. Fig. 3 shows a comparison of growth rate versus error in predicted growth rate for a number of shots on the same experimental day. The model used for comparison here was a multi-filament rigid $\mathrm{z}$ only plasma.
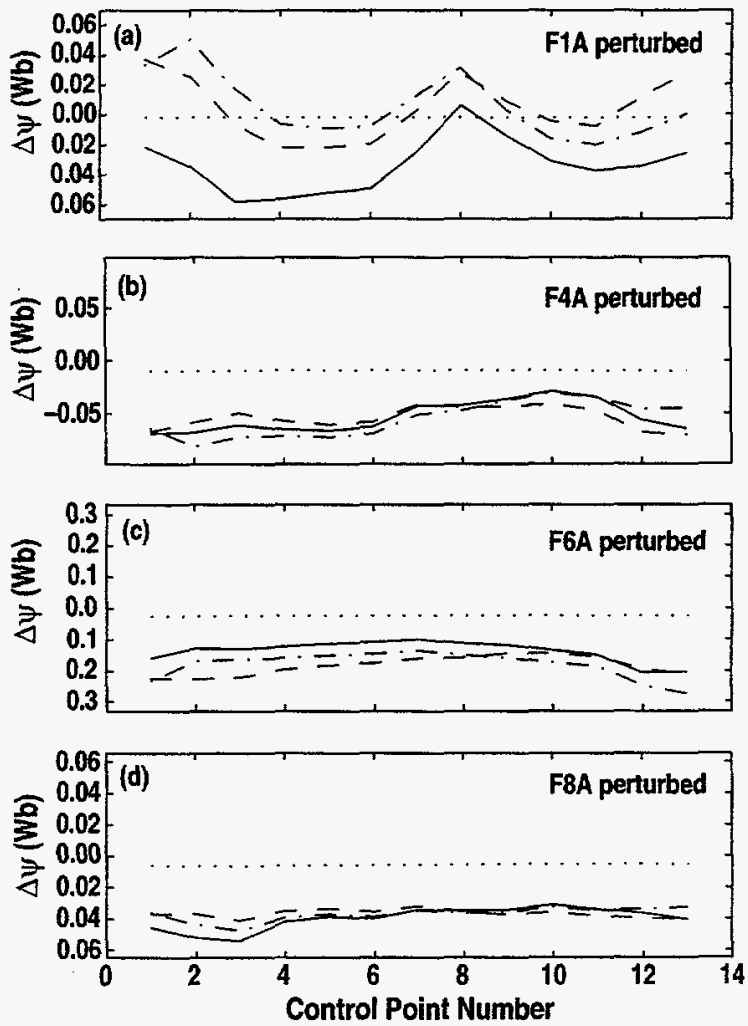

Fig. 2. Comparison of flux change from equilibrium at control points corresponding to equilibrium plasma boundary: solid=actual (EFIT calculated), dash=rigid $\mathrm{r}$ and $\mathrm{z}$ model prediction, dash-dot=rigid $\mathrm{r}$ model prediction. 


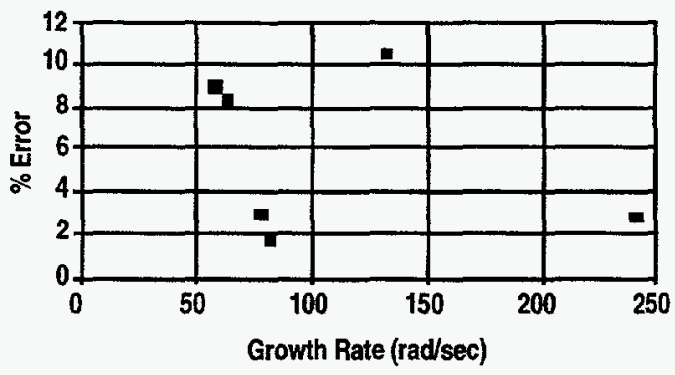

Fig. 3. Scatter plot of growth rate versus error in growth rate prediction.

\section{MULTIVARIABLE CONTROL DESIGN}

Current work which seeks to exploit the new real time equilibrium reconstruction capability and the "isoflux" control approach is the development of true multivariable controllers which can account for the many dependencies of flux at various locations on currents in shaping coils. Our initial work on this problem attempts to understand through simulations the impact of the choice of models on development and implementation of controllers for DIII-D.

The first plasma model used in our simulations was the rigid radial plasma described above. We chose to use the normalized coprime factorization (NCF) [5] design technique to design controllers and to test them in a closed loop mixed linear/nonlinear plasma simulation, shown in Fig. 4. It uses a linearized plant to evolve coil and vessel currents, followed by a nonlinear algorithm which performs the following computations: (1) calculate $B_{r}$ and $B_{z}$ error at the $X$-point, (2) estimate $X$-point location and flux at $X$-point using the same algorithm as in the real time code, (3) compute flux at specified control points. Control of the X-point location in DIII-D experiments using the isoflux technique has been done by feeding back the $\mathrm{X}$-point $\mathrm{r}$ and $\mathrm{z}$ errors directly to a controller. The simulation on the other hand computes a field error $\left(\Delta \mathrm{B}_{\mathrm{r}}, \Delta \mathrm{B}_{\mathrm{z}}\right)$ from the $\mathrm{X}$-point error $(\Delta \mathrm{r}, \Delta \mathrm{z})$ as follows:

$$
\Delta B_{r}=\frac{\partial B_{r}}{\partial r} \Delta r+\frac{\partial B_{r}}{\partial z} \Delta z, \Delta B_{z}=\frac{\partial B_{z}}{\partial r} \Delta r+\frac{\partial B_{z}}{\partial z} \Delta z
$$

and attempts to drive that error to 0 . Here, partials are estimated from field values on the $\mathrm{X}$-point grid (Fig. 1). The flux errors (flux at control points minus calculated $\mathrm{X}$-point flux), $\Delta \mathrm{B}_{\mathrm{r}}$ and $\Delta \mathrm{B}_{\mathrm{Z}}$ at the $\mathrm{X}$-point, and the $\mathrm{F}$-coil currents are fed back to the controller which calculates a voltage demand for the shape control power supplies.

Plasma shape controllers were designed with a plant model which assumed that the flux/field at all points on a control segment (and over the $\mathrm{X}$-point grid) in response to a given conductor current is equal to the average over the segment (grid) of the true responses to that current. This appears to be a reasonable approximation for flux responses, since vacuum flux always increases with increasing current, i.e. the sign of the response remains constant. (Not always true with plasma present however.) However, for certain F-coils, the direction

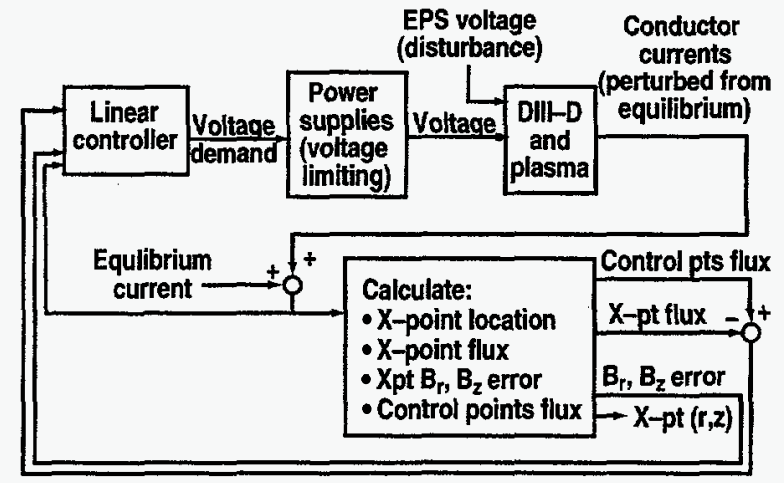

Fig. 4. Closed loop simulation of DIII-D, including plasma, with multivariable controller.

of change in vacuum field at a grid point with increasing current depends on which point on the grid is being measured. The effect of this difference in sign is that the same change in the current in coil F8B (Fig. 1), for example, will sometimes push the $\mathrm{X}$-point to the right and sometimes to the left, depending on where the $\mathrm{X}$-point is initially. In these cases, the model response was artificially set to 0 .

Several controllers were designed and tested in simulation. Those controllers which were designed with knowledge of the plasma model all provided robust closed loop stability and traded off such quantities as response time, voltages used, coil current excursions, and stability robustness as measured by the NCF robustness parameter $\varepsilon$. An example is shown in Fig. 5. Note that $X$-point control has been emphasized at the expense of boundary control because of the impact in DIII-D of the strike point location on the ability to perform certain physics studies. $\mathrm{X}$-point weights also included integral gain to avoid long term offsets in $\mathrm{X}$-point position control.

While voltages remained well below their limits, we see that coil currents are moving to a significantly different equilibrium; in some cases currents are larger. In the controller used, integral gain was intentionally not used for the control point fluxes because of the desire to maintain lower equilibrium F-coil currents by allowing long term offsets in flux at the control points.

Several attempts were also made to design a controller using only the conductors model without the plasma response which would provide "reasonable" control of the crude plasma we are using. All such controllers were very robustly closed loop stable (as measured by $\varepsilon$ ) for the plasmaless system, but most destabilized the initially stable plasma-included plant in simulation. After many iterations, a controller was obtained which gave closed loop stability and performed comparably to the "plasma-model" designed controller. Note however that each design iteration required running the simulation (which includes a plasma model) to see that it worked. Thus we cannot say the controller was developed completely independent of a plasma model. 

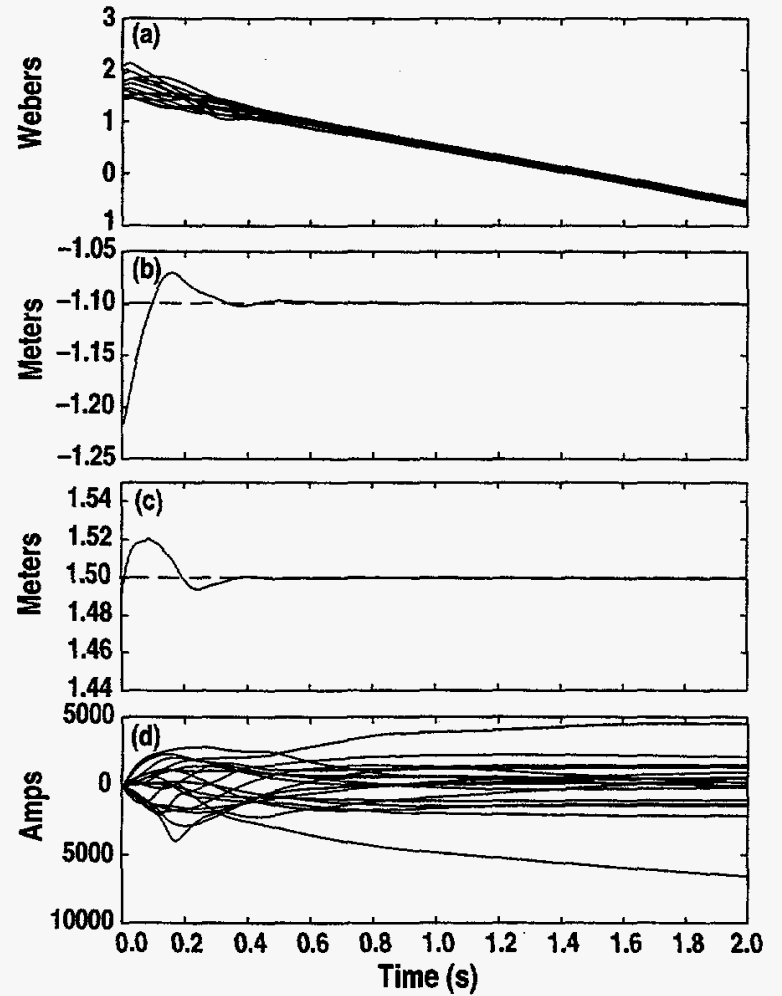

Fig. 5. Closed loop response with controller designed for rigid radial plasma response: (a) measured flux on segment control points (dashed line $=$ flux at X-point), (b) X-point $\times$ coordinate and desired value, (c) $X$-point $z$ coordinate and desired value, (d) F-coil currents

A second simulation used the rigid $\mathrm{r}$ and $\mathrm{z}$ plasma model whose validation was discussed above. Because this plasma is unstable, the DIII-D analog vertical position control algorithm was implemented in the simulation as a seperate control loop to stabilize the system before application of the shaping control. The controller designed using only the rigid $r$ plasma model was used to control the rigid $\mathrm{r}$ and $\mathrm{z}$ plasma system. Results are similar to those shown in Fig. 5 except for a small oscillation in all signals throughout the simulation; this oscillation is driven by the vertical position control loop and is not yet well understood.

\section{SUMMARY}

In this paper, we have shown comparisons of predictions from both linearized single filament plasma models and the DIII-D vacuum model versus experimental data. These preliminary results indicate that the dynamics of the plasma/tokamaksystem (coil and conductor current evolution) are rather insensitive to the plasma model used, while static system responses require some form of plasma model. We have seen that single filament models can provide some capability for prediction of static control parameters, but are not sufficiently accurate for control design. In particular, we have described attempts to design a plasma controller using only knowledge of the vacuum flux and field response. Although a working controller was obtained, it required many "trial-and-error" iterations before it could successfully control a plasma in simulation. This is in contrast to the ease with which a controller was designed when an accurate model of the simulated plasma was available. Multifilament models were found to provide a sufficiently accurate estimate of vertical growth rate, provided external conductors were adequately modeled. The focus of further work will be on extending the single filament plasma model to multifilament models to determine whether adequate predictive capability for shape control can be obtained using only these simple modeling techniques.

\section{REFERENCES}

[1] S. Kinoshita, et al., "Independent control of gaps in single-null divertor discharges on the DIII-D tokamak," General Atomics Report GA-A19584 (1989).

[2] J.R. Ferron, et al., "Real time equilibrium reconstruction for tokamak discharge control," General Atomics Report GA-A22586, (1997), unpublished.

[3] M.L. Walker, A. Nerem, D. Baggest, "Advanced plasma control final report, part I: DIII-D system models for feedback control," General Atomics Report GA-C21745 (1996).

[4] E.A. Lazarus, J.B. Lister, G.H. Nielson, "Control of the vertical instability in tokamaks," Nucl. Fusion 30, 111 (1990).

[5] D.C. McFarlane, K. Glover, "Robust controller design using normalised coprime factor plant descriptions," Springer-Verlag, Lect. Notes in Control and Inf. Sci., Vol 138, 1989. 